Z Herz- Thorax- Gefäßchir 2012 $\cdot 26: 85-85$

DOI 10.1007/s00398-012-0916-8

Online publiziert: 21. März 2012

(c) Springer-Verlag 2012

Klaus Kallenbach

Chirurgische Klinik, Klinik für Herzchirurgie, Universitätsklinikum Heidelberg

\title{
Was hat Motorenentwicklung mit Herzchirurgie zu tun?
}

Liebe Leserinnen und Leser, wer zum ersten Mal mit dieser Ausgabe die Zeitschrift für Herz-, Thorax- und Gefäßchirurgie aufschlägt, dürfte sich wundern. Zunächst findet er im Inhaltsverzeichnis Artikel, die man im offiziellen Weiterbildungsorgan der Deutschen Gesellschaft für Thorax-, Herz- und Gefäßchirurgie erwartet: ein bunter Strauß vielversprechender Artikel aus verschiedenen Rubriken, die sich mit relevanten Themen des Fachs beschäftigen, darunter eine aktuelle Bestandsaufnahme zur kathetergestützten Aortenklappenimplantation (TAVI), Artikel zur temporären Herz- und Lungenunterstützung, zur Verwendung von Einzel- oder Sequenzialvenengrafts, Forschungsergebnisse zur Organkonservierung bei Lungentransplantation, aber auch „weichere" Themen wie die Beiträge zur empathischen Führung oder zu Stressoren am Arbeitsplatz Klinik werden adressiert. Und ganz am Ende des Verzeichnisses dann das: unter der Rubrik „Blick über den Tellerrand“ ein Artikel vom Motorenentwickler Prof. F. Indra zur (relevanten) Frage, womit wir morgen unsere Autos bewegen. Was hat das mit Herzchirurgie zu tun?

Ganz ehrlich: Nichts! Jeder Versuch, hier eine künstliche Verbindung zum Fach herzustellen, beispielsweise über die wissenschaftliche Aufarbeitung eines Themas, wirkte stümperhaft. Auch ist die Darstellung des Autors kaum mit Literaturverweisen belegt, vielmehr stellt der Artikel die Meinung eines bekannten, erfahrenen Motorenbauers dar, der Jahrzehnte erfolgreich für die Automobilindustrie gearbeitet hat. Insofern repräsentiert der Inhalt des Beitrags nicht die Meinung der Herausgeber. Interessant, vielleicht auch polarisierend ist dessen negative Beurteilung des Elektroautomobils, die man so nicht erwartet hätte. Lobbyismus? Ich erlaube mit in diesem Falle kein Urteil, dazu fehlt das
Hintergrundwissen. Bei der Beschäftigung mit dem Thema, welches sicherlich auch eine politische Dimension hat, stieß ich jedoch auf ein soeben für das Bundesumweltministerium erstelltes „Hintergrundpapier Elektromobilität" aus dem ÖkoInstitut e. V. (www.oeko.de). Auch hier kommt das Elektroauto in Sachen Klimabilanz schlecht weg; wesentliche Thesen Friedrich Indras werden bestätigt.

Warum wir diesem Thema in unserer Zeitung Raum geben? Weil wir Sie nicht nur weiterbilden, sondern auch auf hohem Niveau unterhalten möchten! Dieser Artikel soll Sie durchaus zerstreuen und dennoch informieren, Sie auch „einfangen“, indem Sie als Leser mit einem leicht anmutenden Einstieg über ein aktuelles, allgemein interessierendes Thema an die schwerere Kost der Fachartikel herangeführt werden. Vielleicht funktioniert dies auch im Nachtdienst, wenn zwischen 3 und $5 \mathrm{Uhr}$ etwas Ruhe einkehrt, und Sie die Zeitschrift zum „Schmökern“ aufschlagen. Und wenn Sie dann doch auch einen Weiterbildungsartikel lesen, ist unser Ziel bereits erreicht!

Ihnen, liebe Leserinnen und Leser, wünsche ich viel Spaß beim Lesen, Lernen und Schmökern!

Herzliche Grüße,

Ihr

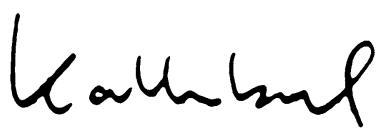

Klaus Kallenbach

\section{Korrespondenzadresse}

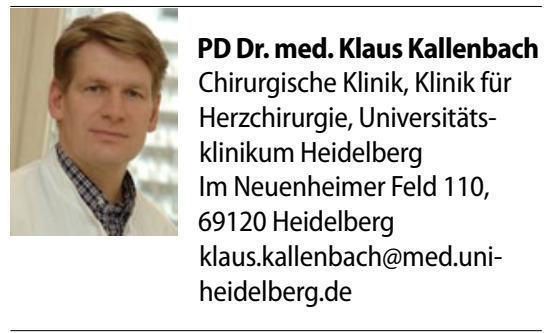

